\title{
Perfil dos pacientes com perdas funcionais e dependência atendidos pelo PSF no município de São Paulo*
}

\author{
PROFILE OF THE PATIENTS WITH FUNCTIONAL LOSSES AND DEPENDENCE IN \\ THE FAMILY HEALTH CARE PROGRAM IN SÃO PAULO \\ PERFIL DE LOS PACIENTES CON PERDIDAS FUNCIONALES Y DEPENDENCIA
ATENDIDOS POR EL PSF EN EL MUNICIPIO DE SÃO PAULO
}

\section{Jaqueline Correia Gaspar', Maria Amélia de Campos Oliveira", Maria de Fátima Faria Duayer'II}

\section{RESUMO}

Buscou-se compreender como as desigualdades sociais e de saúde expressam-se no perfil saúdedoença de pessoas, com perdas funcionais e dependência, atendidas no domicílio por equipes de Saúde da Família nos distritos administrativos do município de São Paulo. Os distritos foram agrupados em relação ao Índice de Exclusão Social por meio de uma análise de cluster, seguida da descrição estatística das variáveis, comparando-as entre si. Para o município como um todo, verificou-se predominância de mulheres idosas, com incapacidade leve e que requerem cuidados de menor complexidade, compatíveis com a Atenção Básica. Nos distritos com maior exclusão social, identificou-se proporção de homens com menos de 60 anos e crianças com incapacidade severa, que necessitam de cuidados de maior complexidade em relação aos de menor exclusão. Discute-se a necessidade de elaboração de políticas de atenção domiciliária que contemplem as especificidades do município de São Paulo em substituição àquelas focadas em grupos populacionais específicos.

\section{DESCRITORES}

Assistência domiciliar.

Atenção primária à saúde.

Condições sociais.

Perfil de saúde.

\section{ABSTRACT}

The aim of this study was to understand how social and health inequalities are expressed in the health-disease profile of individuals with functional losses and dependence receiving home care by Family Health Care Program's teams in the administrative districts of the city of São Paulo. The districts were grouped according to the Social Exclusion Index through a cluster analysis, and a statistical description of the variables was developed in order to compare them. For the city as a whole was verified the prevalence of senior women with light functional losses and dependence requiring low complexity care, compatible with Primary Health Care. Districts with major social exclusion had a larger proportion of males less than 60 years old and children with severe disabilities who need care of greater complexity. The article debates the need for home care policies designed for the specificities of the city of São Paulo instead of policies focused on specific population groups.

\section{KEY WORDS}

Home nursing.

Primary health care.

Social conditions.

Health profile.

\section{RESUMEN}

Se buscó comprender como las desigualdades sociales y de salud se expresan en el perfil saludenfermedad de personas, con pérdidas funcionales y dependencia, atendidas en el domicilio por equipos de Salud de la Familia en los distritos administrativos del municipio de São Paulo. Los distritos fueron agrupados en relación al Índice de Exclusión Social por medio de un análisis de cluster, seguida de la descripción estadística de las variables, comparándolas entre sí. Para el municipio como un todo, se verificó predominancia de mujeres ancianas, con incapacidad leve y que requieren cuidados de menor complejidad, compatibles con la Atención Básica. En los distritos con mayor exclusión social, se identificó proporción de hombres con menos de 60 años y niños con incapacidad severa, que necesitan de cuidados de mayor complejidad en relación a los de menor exclusión. Se discute la necesidad de elaboración de políticas de atención domiciliaria que contemplen las especificidades del municipio de São Paulo en sustitución aquellas focalizadas en grupo poblacionales específicos.

\section{DESCRIPTORES}

Atención domiciliaria de salud.

Atención primaria de salud.

Condiciones sociales.

Perfil de salud.
* Extraído da dissertação "Quem precisa de cuidados no domicílio? Investigando os perfis das pessoas atendidas por equipes de Saúde da Família no município de São

Paulo", Escola de Enfermagem, Universidade de São Paulo, 2006.

I Enfermeira. Mestre em Enfermagem em Saúde Coletiva pela Escola de Enfermagem,Universidade de São Paulo (EEUSP), São Paulo, SP, Brasil. jaqueline.cgaspar @gmail.com

II Enfermeira. Professora Livre-Docente do Departamento de Enfermagem em Saúde Coletiva, Escola de Enfermagem, Universidade de São Paulo (EEUSP), São Paulo, SP, Brasil. macampos@usp.br

III Médica Sanitarista. Coordenadora da Atenção Básica do Programa de Saúde da Família, Secretaria Municipal de Saúde de São Paulo, São Paulo, SP, Brasil. fadua@uol.com.br 


\section{INTRODUÇÃO}

Face às transições demográfica e epidemiológica observadas em nosso país nas últimas décadas, muitos autores têm discutido as alterações nos perfis de saúde-doença das populações e suas implicações para a atenção à saúde.

O crescimento demográfico observado entre as décadas de 40 e 70 foi resultado do rápido declínio das taxas de mortalidade, principalmente a infantil, e do aumento das taxas de natalidade e da esperança de vida, alterando o ritmo de crescimento populacional e resultando, em um primeiro momento, em rejuvenescimento da população ${ }^{(1-2)}$. O processo de envelhecimento no Brasil só teve início a partir da década de 60 , quando houve redução efetiva da fecundidade, com queda no ritmo de crescimento anual do número de nascimentos e progressivo estreitamento da pirâmide etária da população.

Quando as crianças nascidas a partir da década de 70 alcançaram o período reprodutivo, a queda da fecundidade resultou no estreitamento da base da pirâmide populacional. Neste momento em que as coortes extensas da década de 40 ultrapassaram os 60 anos, observa-se o incremento progressivo do número de idosos, com tendência a se acentuar nos próximos anos.

O impacto social causado pela velocidade com que se processaram as mudanças demográficas traduz-se em grandes dificuldades para o Estado brasileiro, que precisa lidar com um novo perfil epidemiológico em que predominam doenças crônico-degenerativas, sem que se substitua o anterior, em que predominavam as doenças infecto-contagiosas, caracterizando uma polarização epidemiológica ${ }^{(2)}$.

Há uma relação direta entre os processos de transição demográfica e epidemiológica, pois, ao serem beneficiados pela diminuição da mortalidade por doenças infecto-contagiosas, os grupos mais jovens da população sofrem outros desgastes que favorecem o aparecimento de doenças crônico-degenerativas.

No Brasil, o aumento do número de anos de vida não se traduz necessariamente em uma velhice saudável. As pessoas envelhecem de forma heterogênea de acordo com sua classe social, religião, etnia ou gênero, sendo identificadas como inativas ou improdutivas, sofrendo com baixas pensões e aposentadorias e dificuldade de acesso aos equipamentos públicos em geral, principalmente aos de saúde ${ }^{(3)}$. Deste modo,

[...] estudar o processo de envelhecimento torna-se uma tarefa complexa que requer a particularização de condições, de necessidades, de exigências, de interesses e de possibilidades, de acordo com a classe social, o gênero, a etnia do idoso(4).
Com o envelhecimento populacional, uma das preocupações passa a ser quem cuidará dessa população crescente e a como serão vividos os anos ganhos com o aumento da expectativa de vida?(5). É possível encontrar relação entre a longevidade e as deficiências e incapacidades, indicando que a população, embora vivendo mais, apresenta um grau crescente desses problemas ${ }^{(6)}$.

Um estudo realizado na década de 90 no município de São Paulo com a finalidade de caracterizar o perfil do idoso revelou que $86 \%$ dos entrevistados referia pelo menos uma doença crônica, $47 \%$ requeria ajuda parcial ou total para realizar pelo menos uma das atividades cotidianas e 7\% necessitava de ajuda para realizar sete ou mais atividades da vida diária, demandando assistência constante e relativamente especializada. O ônus do cuidado dessas pessoas tem recaído sobre as famílias, ocultando uma importante demanda por políticas sociais e de saúde. Numa família com parcos recursos econômicos, cuidar de um idoso dependente pode se tornar insustentável ${ }^{(7)}$.

Em São Paulo, as mulheres idosas vivem mais tempo com incapacidade e dependência que os homens. A partir dos 60 anos, as mulheres podem esperar viver mais 22,2 anos e desses, 2,5 (11,2\%) com incapacidade e dependência. Após os 60 anos, os homens podem chegar a viver mais 17,6 anos, 1,6 (9,1\%) dos quais com incapacidade e dependência ${ }^{(5)}$.

Para melhor compreender os determinantes da perda de funcionalidade e, conseqüentemente, da dependência, é preciso examinar fenômenos a ela associados, representados não apenas pelo envelhecimento populacional, mas também pelas conseqüências do aumento da violência. Faz-se necessário, portanto, compreender como a população está envelhecendo no Brasil e a repercussão deste fenômeno nos serviços de saúde, bem como apreender os determinantes estruturais que atuam sobre as formas de envelhecer e que também promovem o aumento da exclusão social e da violência.

Nas grandes cidades também é digno de nota o expressivo incremento da violência urbana, expressão das desigualdades impostas pela vigência do sistema capitalista em nosso país, resultando em mortes prematuras de pessoas jovens, principalmente homens em idade produtiva. Os que sobrevivem aos acidentes por armas de fogo ou de trânsito, não raro apresentam mutilações ou deficiências físicas com limitações importantes.

O perfil epidemiológico brasileiro reside num emaranhado de iniqüidades sociais com origens estruturais, políticas e econômicas, de modo que as diferentes formações sociais apresentam perfis de saúde-doença que variam de acordo com o desenvolvimento das forças produtivas e das rela- 
ções sociais de produção(8-9). O processo de exclusão social no Brasil agravou-se após a instauração das chamadas políticas de ajuste de caráter neoliberal, adotados no contexto da globalização que, em conjunto com a exclusão, são conceitos que definem realidades interligadas. Se, por um lado, a globalização representa o momento do desenvolvimento do capitalismo mundial, por outro a exclusão exprime as conseqüências imediatas deste processo ${ }^{(10)}$.

A população, cada vez mais excluída pelas políticas de ajuste, tem apenas o Estado como garantia de salvaguarda de seus direitos sociais. No entanto, investimentos voltados para as áreas de bem estar social são feitos de forma irregular e descontínua. A globalização não é equilibrada nem harmônica e, ao invés de atenuar as disparidades de renda, amplifica-as ${ }^{(10)}$.

A relação entre políticas sociais e pobreza no Brasil tem grande importância. A população mais afetada pela deterioração das políticas e programas públicos e sociais é predominantemente carente ou excluída. O Estado brasileiro, na tentativa de fazer parte do seleto mundo desenvolvido, na maior parte das vezes acaba por aceitar as normas internacionais de ajuste e redução de gastos, adotando estratégias focalizadas e pouco resolutivas, fazendo com que a exclusão assuma maiores proporções.

O agravamento das condições sociais da população leva ao aumento da demanda por programas sociais que sofrem continuamente com as restrições orçamentárias e a descontinuidade de repasse dos recursos estatais ${ }^{(11)}$. De um lado, há o desemprego estrutural e tecnológico, sob uma política de lucratividade que acaba por transformar os trabalhadores em lixo industrial e, de outro, o desmantelamento da legislação de amparo ao trabalhador ${ }^{(12)}$.

O endividamento público e a abertura dos mercados dentro do processo de globalização reduziram a capacidade do Estado em combater a exclusão social. Se antes, em um ambiente de maior ocupação da população assalariada, as políticas econômicas e sociais eram capazes de garantir a universalização do acesso à saúde, educação, aposentadoria e moradia, hoje, quando se observa taxa de desemprego recorde na história do país, estas estratégias não mais são suficientes: a exclusão passa agora por pessoas que já foram incluídas um dia ${ }^{(13)}$.

\section{O MUNICÍPIO DE SÃO PAULO}

Os diversos estudos que tratam o panorama da exclusão social no município de São Paulo mostram que a cidade guarda peculiaridades intra-regionais que dificultam sua governabilidade. Nas subprefeituras, os diferentes distritos apresentam realidades extremamente díspares. Segundo o Atlas da Exclusão Social, nas áreas sul e leste da capital, mais de $30 \%$ dos 96 distritos administrativos podiam ser considera- dos como de elevada exclusão social (Índice de Exclusão Social menor que 0,4 ). Nessas regiões vive $47 \%$ da população paulistana, sendo que $40 \%$ são jovens com até 19 anos de idade, que representam $54,8 \%$ de todos os jovens do município. Estas regiões também são responsáveis pelos $62,3 \%$ dos analfabetos da cidade. A escolaridade média dos chefes de família é de 6,6 anos, contra os 8,5 anos verificados para a Capital como um todo ${ }^{(14)}$.

Os sinais e sintomas da pobreza e da exclusão social na cidade não podem mais ser ignorados. Vêem-se, por todos os lados, crianças trabalhando nos semáforos, o crescimento assustador da violência e o aumento indiscriminado de pessoas vivendo na mais severa pauperização. Muitos ainda vêem a pobreza como sinal de atraso e, portanto, um pesado resíduo do passado a ser enfrentado no presente, ao invés de a entender como estrutural e estruturante da sociedade brasileira ${ }^{(13)}$.

\section{ASSISTÊNCIA DOMICILIÁRIA}

Para os que sobrevivem às doenças crônicas e degenerativas e à violência, muitas vezes com seqüelas que dificultam a locomoção e o acesso aos serviços de saúde, a assistência domiciliária representa uma alternativa possível para assegurar o direito e a eqüidade na assistência à saúde.

O cuidado domiciliário ressurge como uma das possibilidades de promover mudanças na qualidade da atenção à saúde, pautado na reversão do modelo assistencial centrado no hospital e no profissional médico, privilegiando a construção de uma nova lógica de atenção, com enfoque na promoção e prevenção à saúde e na humanização da assistência. Embora represente uma boa alternativa para pessoas com perdas funcionais e dependência, os serviços públicos de saúde ainda não estão estruturados adequadamente para dar assistência domiciliária integral ao paciente, sua família e cuidadores, familiares ou não ${ }^{(15)}$.

Em pessoas com incapacidade, o processo saúde-doença é marcado pela prevalência de múltiplos problemas de saúde, demandando articulação em diferentes níveis de atenção. Para os quadros agudos, o tratamento hospitalar faz-se necessário, mas, para os casos estáveis, o acompanhamento habitual pode ser realizado no domicílio por equipes de Atenção Primária ${ }^{(16)}$.

Atualmente, uma das principais dificuldades enfrentadas pelo setor saúde é determinar quem necessita de cuidados no domicílio e identificar as necessidades de saúde dessas pessoas. A estratégia Programa Saúde da Família, que realiza a vigilância à saúde na área de abrangência das Unidades Básicas e trabalha com população adstrita, traz a possibilidade de melhor identificar o perfil de morbidade das famílias sob sua responsabilidade por meio do cadastramento dos moradores do território. Por conseguinte, permite iden- 
tificar o perfil dos pacientes que necessitam de algum tipo de cuidado domiciliário.

O presente estudo buscou compreender como a população que necessita de cuidados domiciliários de saúde está distribuída no município de São Paulo e as desigualdades sociais e de saúde associadas a essa distribuição, tendo por finalidade oferecer subsídios para a formulação de políticas públicas voltadas para as necessidades de saúde das pessoas com perdas funcionais e dependência que levem à reordenação da assistência à saúde na Atenção Básica.

\section{MÉTODO}

Realizou-se um estudo epidemiológico não-experimental de caráter transversal e descritivo, do tipo ecológico e de base territorial, para descrever, por meio de dados secundários, os perfis das pessoas com perdas funcionais e dependência atendidas em domicílio por equipes de Saúde da Família no município de São Paulo em julho de 2003.

A amostra constituiu-se de 7.160 pessoas, atendidas em 111 Unidades de Saúde da Família (USFs) distribuídas de forma heterogênea em 45 distritos administrativos da capital. Os dados foram processados e analisados por meio dos softwares SPSS $^{\circledR}$ 13.0, EPI-INFO 2000 versão 3.3.2 e Excell 8.0 ${ }^{\circledR}$.

Os distritos administrativos pesquisados foram agrupados por similaridade em relação ao Índice de Exclusão Social para o município de São Paulo ${ }^{(14)}$. Este índice varia entre 0 e 1 , sendo que quanto mais próximo de 0 , pior a situação de exclusão social. Os 96 distritos administrativos foram analisados por meio dos indicadores pobreza, emprego, desigualdade, alfabetização, escolaridade, juventude e violência que, agrupados, compõem o Índice de Exclusão Social (Iex) de cada um dos distritos administrativos.

O agrupamento dos distritos foi feito por meio de uma análise de cluster utilizando o método da variância mínima, com agrupamento hierárquico aglomerativo e distância euclidiana ao quadrado(a) . A análise resultou em quatro grupos nomeados de acordo com a sua situação de exclusão em: Exclusão alta (Iex entre 0,231 e 0,302), Exclusão moderada (Iex entre 0,323 e 0,441), Exclusão baixa (Iex entre 0,459 e 0,551) e Exclusão muito baixa (Iex entre 0,611 e 0,800).

Em seguida, procedeu-se a descrição estatística das variáveis: número de pacientes por distrito, idade (em anos), sexo, diagnóstico (lista de morbidade do CID-10) ${ }^{(17)}$, grau de incapacidade e indicador de complexidade de cuidado $^{(\mathrm{b})}$ por

(a) Distância euclidiana: medida de distância obtida pela aplicação do Teorema de Pitágoras.

(b) A complexidade de cuidado foi medida pelo uso de oxigenoterapia domiciliar, presença de traqueostomia, presença de úlcera por pressão e/ou uso de sonda. Foram considerados de maior complexidade de cuidado àqueles que apresentassem pelo menos um destes indicadores. grupo, comparando-os entre si. O grau de incapacidade representa uma síntese do Escala de Avaliação da Incapacidade Funcional da Cruz Vermelha Espanhola, que estabelece seis graus assim definidos: Grau 0: Vale-se totalmente por si mesmo. Caminha normalmente; Grau 1: Realiza suficientemente as Atividades da Vida Diária (AVD). Apresenta algumas dificuldades para locomoções complicadas; Grau 2: Apresenta algumas dificuldades nas AVD, necessitando apoio ocasional. Caminha com ajuda de bengala ou similar; Grau 3: Apresenta graves dificuldades nas AVD, necessitando de apoio em quase todas. Caminha com muita dificuldade, ajudado por pelo menos uma pessoa; Grau 4: Impossível realizar, sem ajuda, qualquer das atividades de vida diária. Capaz de caminhar com extraordinária dificuldade, ajudado por pelo menos duas pessoas; Grau 5: Imobilizado na cama ou sofá, necessitando de cuidados contínuos. O grau de incapacidade leve agrupa os graus 1 e 2, o grau moderado corresponde ao grau 3 e o grau severo de incapacidade corresponde aos graus 4 e 5 .

Verificou-se a existência de correlação entre as variáveis e o Índice de Exclusão Social por meio dos coeficientes de Pearson e Spearman, após aplicação do teste de normalidade de Kolmorogorov-Smirnov. Procedeu-se o teste de significância das eventuais correlações encontradas, adotando a significância de 0,05.

Os achados foram discutidos à luz da Determinação Social do Processo Saúde-Doença, utilizando-se a metodologia proposta pela Teoria da Intervenção Práxica de Enfermagem em Saúde Coletiva - TIPESC ${ }^{(18)}$ em que,

na sua vertente metodológica, é a sistematização dinâmica de captar e interpretar um fenômeno articulado aos processos de produção e reprodução social referentes à saúde e doença de uma dada coletividade, no marco de sua conjuntura e estrutura, dentro de um contexto social historicamente determinado...(18)

Operacionalmente, a teoria propõe cinco etapas: a) captação e b) interpretação da realidade objetiva; c) construção do projeto de intervenção; d) intervenção e e) reinterpretação da realidade objetiva. O presente estudo deteve-se na captação e interpretação da realidade objetiva nas dimensões estrutural (formação social e estruturação da sociedade), particular (perfis epidemiológicos dos grupos sociais) e singular (manifestações do processo saúde-doença) ${ }^{(18)}$.

\section{RESULTADOS}

A análise de cluster resultou em quatro grupos de distritos similares entre si, como se segue. 
Quadro 1 - Distribuição dos distritos em quatro grupos homogêneos, após aplicação das técnicas de agrupamento escolhidas - Município de São Paulo - 2003

\begin{tabular}{|c|c|c|c|c|c|c|c|}
\hline \multicolumn{2}{|c|}{ Exclusão alta } & \multicolumn{2}{|c|}{ Exclusão moderada } & \multicolumn{2}{|c|}{ Exclusão baixa } & \multicolumn{2}{|c|}{ Exclusão muito baixa } \\
\hline$N^{o} d o D A$ & Nome do Distrito & $N^{o} d o D A$ & Nome do Distrito & $N^{o} d o D A$ & Nome do Distrito & $N^{o}$ do $D A$ & $\begin{array}{c}\text { Nome do } \\
\text { Distrito }\end{array}$ \\
\hline 15 & Iguatemi & 24 & Parque do Carmo & 33 & São Domingos & 5 & Cambuci \\
\hline 20 & Jardim Ângela & 26 & Perus & 43 & Vila Formosa & 32 & Santana \\
\hline 11 & Cidade Tiradentes & 22 & Jardim São Luiz & 3 & Bom Retiro & 2 & Bela Vista \\
\hline 21 & Jardim Helena & 36 & São Miguel & 40 & Tucuruvi & 27 & Pinheiros \\
\hline 16 & Itaim Paulista & 38 & Sapopemba & 17 & Jabaquara & 39 & Saúde \\
\hline 37 & São Rafael & 6 & Campo Limpo & 45 & Vila Matilde & & \\
\hline 23 & Lajeado & 8 & Capão Redondo & 13 & Freguesia do Ó & & \\
\hline 4 & Brasilândia & 9 & Cidade Ademar & 34 & São Lucas & & \\
\hline 14 & Guaianases & 44 & Vila Jacuí & 19 & Jaguará & & \\
\hline 42 & Vila Curuçá & 1 & Artur Alvim & 31 & Sacomã & & \\
\hline \multirow[t]{6}{*}{25} & Pedreira & 30 & Raposo Tavares & 41 & Vila Andrade & & \\
\hline & & 7 & Cangaíba & 28 & Pirituba & & \\
\hline & & 10 & Cidade Líder & 29 & Ponte Rasa & & \\
\hline & & 12 & $\begin{array}{l}\text { Ermelino } \\
\text { Matarazzo }\end{array}$ & & & & \\
\hline & & 18 & $\begin{array}{l}\text { Matarazzo } \\
\text { Jaçanã }\end{array}$ & & & & \\
\hline & & 35 & São Mateus & & & & \\
\hline
\end{tabular}

As USFs distribuem-se de forma heterogênea entre os distritos. O grupo com Exclusão alta tinha média de 3,5 USFs, $\pm 2,5$ desvio-padrão (d.p.). O grupo com Exclusão moderada apresentou média de 2,9 $\pm 2,6$ (d.p.). O grupo com Exclusão baixa teve média de 1,5 $\pm 1,0$ (d.p.) e, finalmente, o grupo com Exclusão muito baixa tinha 1,2 USFs $\pm 0,5$ (d.p). Como a implantação do Programa de Saúde da Família no MSP obedeceu, dentre outros, a critérios de exclusão social, é natural encontrar um número maior de USFs nos distritos que compõem os grupos com maior exclusão. O coeficiente de Spearman entre o número de USFs por DA e o Iex foi de $0,346(p=0,02)$, demonstrando que quanto maior a exclusão social, maior o número de USFs.

Dos 7.160 pacientes, 2.003 (28,0\%) foram localizados no grupo de distritos com Exclusão alta, 2.701 (37,7\%) no

de Exclusão moderada, 2.023 (28,3\%) naquele com Exclusão baixa e 433 (6,0\%) no de Exclusão muito baixa. Houve correlação negativa significativa entre o Iex e o número de pacientes por distrito, sugerindo haver mais pacientes atendidos em domicílio nos distritos de maior exclusão social. Cabe ressaltar mais uma vez que nessas regiões há mais USFs, o que pode influenciar o número de pacientes atendidos.

Em relação à idade, obteve-se média de 63, moda de 79 e mediana de 70 anos. A menor idade encontrada foi de um ano e a maior, 114 anos, $\pm 23,4$ (d.p.). Predominam adultos com mais de 51 anos (75\%). A razão idoso/não idoso foi de 1,9 (calculada a partir da divisão do número de idosos, 60 anos e mais, número de não idosos, menos 1 a 59).

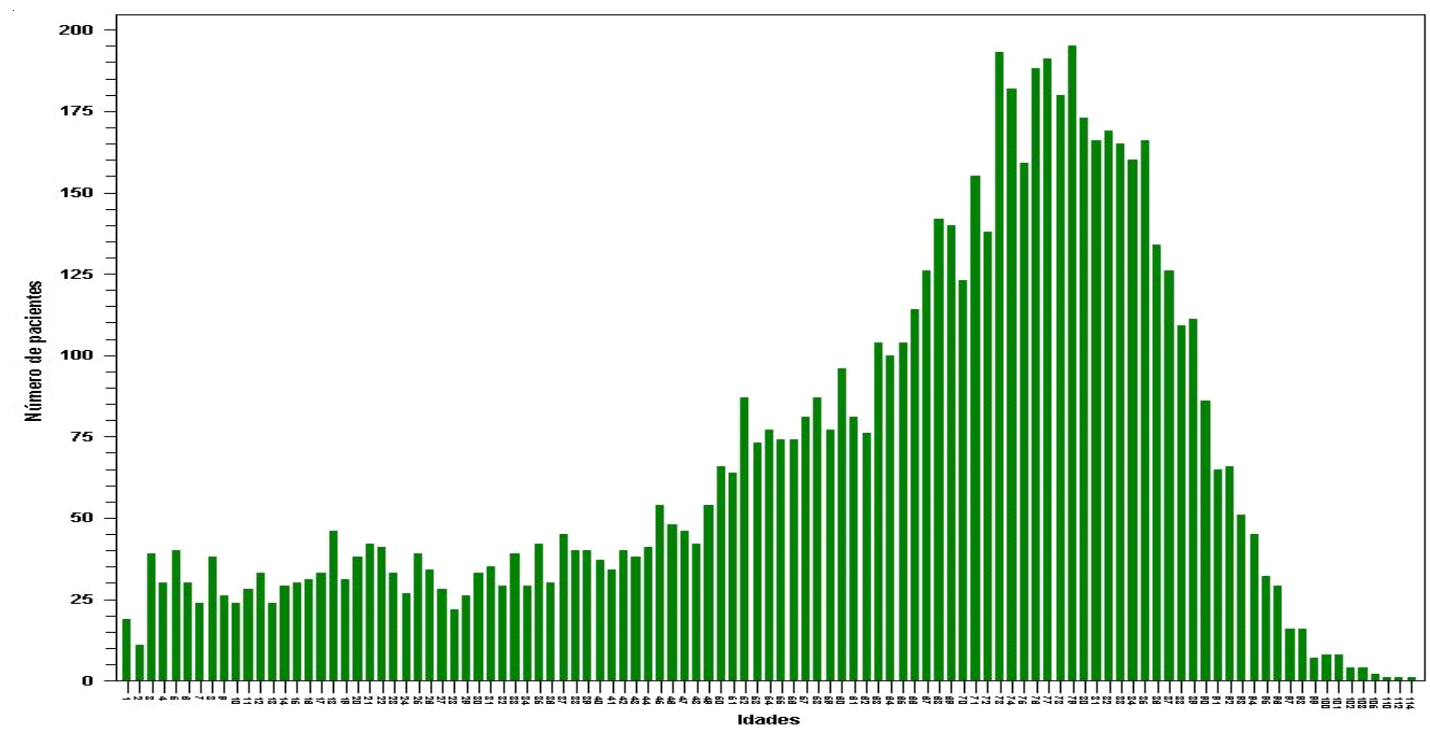

Figura 1 - Distribuição de pessoas de 1 a 114 anos com algum grau de incapacidade - Município de São Paulo - 2003 
Em todos os grupos predominam idosos. Porém, no de maior exclusão, observou-se proporcionalmente mais adultos e crianças. A razão idoso/ não idoso no grupo com $E x$ clusão alta foi de 1,3; no de Exclusão moderada, 3,4; no de Exclusão baixa, 1,8 e no de Exclusão muito baixa, 2,4; destacando-se o grupo de maior exclusão como o que tinha, proporcionalmente, menos idosos. O cálculo da correlação de Pearson entre o a razão idoso/não idoso e o Iex não resultou correlação significativa.

Dentre os 7.160 pacientes estudados, encontrou-se 4.312 (60,2\%) mulheres e 2.848 (39,8\%) homens, numa razão de sexo de 0,66 (foi obtida dividindo-se o total de pessoas do sexo masculino pelo total de pessoas do sexo feminino). Em todos os grupos há mais mulheres que homens. Porém, quanto maior a exclusão, maior a proporção de pessoas do sexo masculino. O grupo com Exclusão muito baixa apresentou razão de sexo de 0,45, enquanto o grupo com Exclusão baixa, razão de 0,57. Já o de Exclusão moderada teve razão de sexo de 0,69 e o grupo com Exclusão alta, 0,77. O coeficiente de correlação de Pearson não demonstrou correlação significativa entre a razão de sexo e o Iex.

Até os 39 anos, a freqüência de homens é o dobro da freqüência de mulheres, em cada faixa etária. A partir dos 60 anos as mulheres passam a ser maioria. Do total de mulheres, $73 \%$ são idosas. Entre os homens, 54,8\% são idosos. A Figura 2 ilustra essa distribuição.

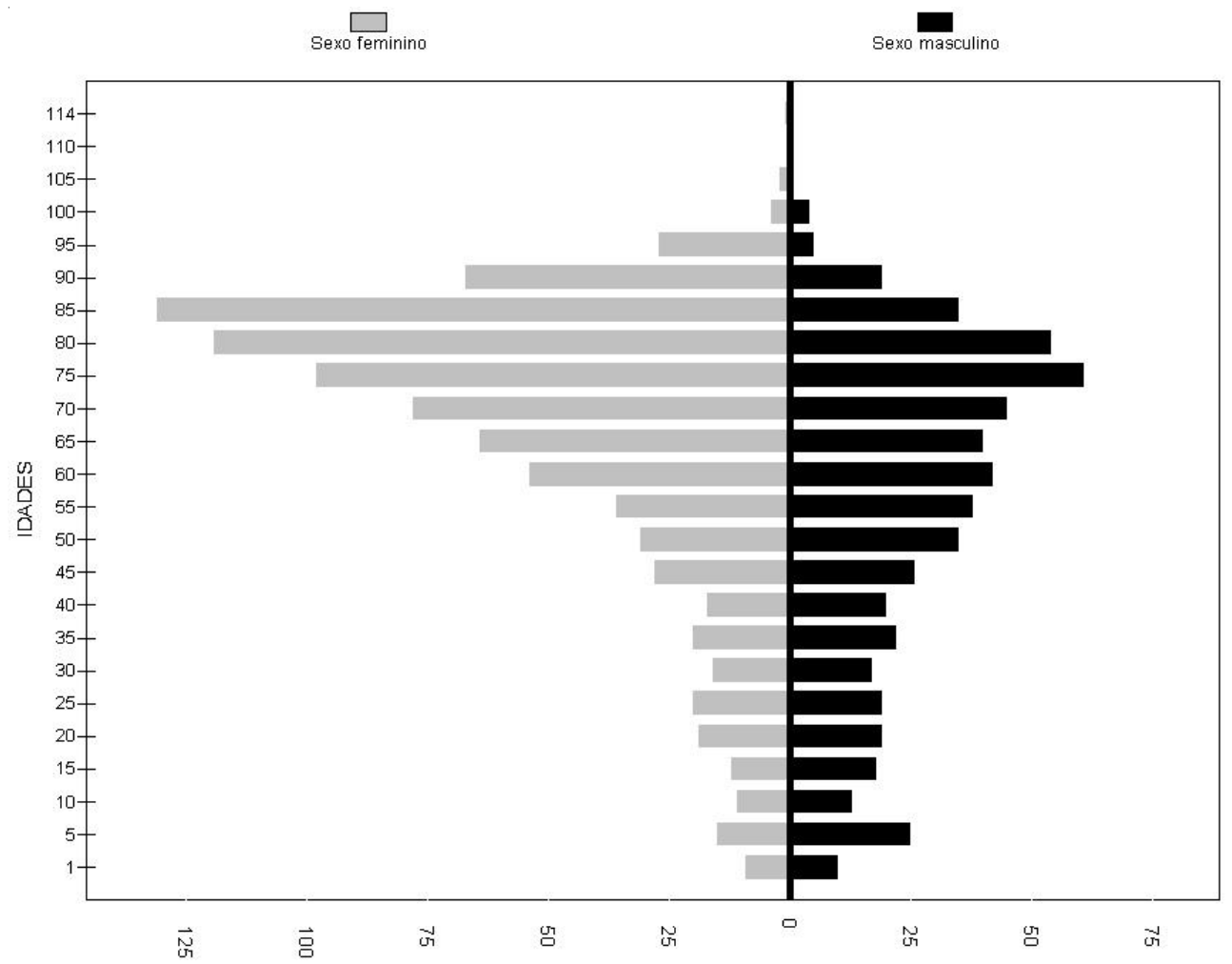

Figura 2 - Pirâmide etária de distribuição das pessoas com algum grau de incapacidade, segundo sexo - Município de São Paulo - 2003

A partir dos 60 anos, a distribuição das pessoas em relação ao sexo é bastante desproporcional. Até os 75 há crescimento, ainda que diferenciado, no número de pessoas com incapacidade em ambos os sexos. A partir daí, inicia-se o decréscimo no número de homens e o crescimento da participação das mulheres mantém-se. Aos 85 anos, a diferença entre os gêneros é a mais expressiva: para pouco mais de 25 homens há mais de 125 mulheres, ou seja, aproximadamente cinco mulheres para cada homem. Em geral, é mais provável encontrar homens que mulheres até os 40 anos, principalmente nos grupos com Exclusão alta, moderada e muito baixa. Nas outras faixas etárias, a predominância é feminina. Apesar da proporção entre homens e mulheres variar de acordo com a exclusão social, o exame do coeficiente de correlação de Pearson entre a razão de sexo e o Índice de exclusão por distrito não mostrou correlação com significância estatística.

As doenças do aparelho circulatório e do sistema nervoso totalizaram $50 \%$ dos diagnósticos, respondendo, respectivamente, por 28\% e 22\% deles. Entre as mais freqüentes estão os acidentes vasculares cerebrais (AVCs) transitórios e síndromes correlatas e os não especificados, como hemorrágico ou isquêmico (22\%), a hipertensão essencial primária (9,4\%), e as paralisias cerebrais (PC) e outras síndromes paralíticas (5,3\%), além de doenças endócrinas, nutricionais e metabólicas, representadas pelo diabetes mellitus (DM), com (8,3\%). Houve ainda 7\% de pessoas clas- 
sificadas como senis e atendidas em decorrência do processo de envelhecimento.

Quanto maior a exclusão social, maior a porcentagem de pessoas acometidas por AVCs e PCs. Por outro lado, quanto menor a exclusão, maior a quantidade de pessoas com hipertensão, diabetes mellitus e senilidade, sugerindo que os perfis de saúde-doença dependem das condições de vida e da situação de exclusão social das coletividades.

A distribuição das doenças entre os sexos mostra que, apesar de os AVCs serem os principais responsáveis pelo atendimento domiciliário, entre os homens, as paralisias cerebrais representam a segunda doença de maior freqüência, enquanto que, para as mulheres o segundo lugar é ocupado pela hipertensão. Em relação às idades, a PC é a doença mais freqüente até os 39 anos de idade.

Em relação aos graus de incapacidade, $47 \%$ das pessoas foram classificadas com incapacidade de grau leve. Porém, $53 \%$ foram consideradas com incapacidade moderada e se- vera, com importantes limitações para se locomover e cuidar de si próprio sem ajuda.

Em todos os grupos, apesar de a maioria das pessoas apresentar incapacidade de grau leve e moderado, os homens apresentavam maior severidade de incapacidade que as mulheres. A maior diferença entre os sexos foi no grupo com Exclusão moderada: a razão de incapacidade para as mulheres era de 0,54 , enquanto que, para os homens, foi de 0,86 (obtida dividindo-se o somatório de pessoas com os graus 4 e 5 pelo somatório de pessoas com os graus 1, 2 e 3). A menor diferença foi observada no grupo com Exclusão baixa, em que a razão de incapacidade encontrada para o sexo feminino foi de 0,21 e, para o sexo masculino, de 0,38.

A hipertensão foi a patologia mais freqüente entre as pessoas com graus leve e moderado de incapacidade, seguida pela senilidade e pelo $\mathrm{DM}$. Nas incapacidades severas, o AVC foi a patologia mais freqüente, seguido pelo DM e PC, respectivamente, como mostra a tabela a seguir.

Tabela 1 - Primeira e segunda patologia de maior freqüência para cada grau de gravidade - Município de São Paulo - 2003

\begin{tabular}{|c|c|c|c|c|c|c|c|}
\hline \multicolumn{2}{|c|}{ Grau de incapacidade } & \multicolumn{6}{|c|}{ Doenças mais freqüentes } \\
\hline & & $\mathbf{1}^{\mathbf{o}}$ & $n_{i}$ & $\% *$ & $2^{\circ}$ & $n_{i}$ & $\% *$ \\
\hline \multirow{2}{*}{ Leve } & Grau 1 & Hipertensão & 192 & 14,5 & Senilidade & 137 & 10,3 \\
\hline & Grau 2 & Hipertensão & 169 & 13,0 & Diabetes mellitus & 144 & 11,1 \\
\hline Moderada & Grau 3 & Hipertensão & 116 & 10,3 & Diabetes mellitus & 110 & 9,8 \\
\hline Severa & Grau 4 & AVC & 197 & 30,8 & Diabetes mellitus & 63 & 9,8 \\
\hline
\end{tabular}

*calculado considerando apenas as pessoas que dispunham de diagnóstico em cada grau.

Entre os 7.160 pacientes pesquisados, $111(1,6 \%)$ pessoas faziam uso de oxigenoterapia, 285 (4,0\%) tinham úlcera por pressão, $86(1,2 \%)$ tinham algum tipo de sonda e 65 (0,9\%) usavam traqueostomia, totalizando 547 (7,6\%) pessoas que apresentavam algum dos indicadores de complexidade de cuidado. De modo geral, nota-se que nas faixas etárias mais elevadas cresce a complexidade do cuidado requerido. Foram encontrados quase dois idosos para cada não idoso entre os que faziam uso de oxigenoterapia e/ou apresentavam úlcera por pressão. Entre os que usavam algum tipo de sonda, os idosos também eram maioria, com pouco menos de dois idosos para cada não idoso. Porém, entre os que usavam traqueostomia, a maioria era de pessoas com até 59 anos.

Apesar de os idosos deterem 62,2\% dos indicadores de complexidade de cuidado, para alguns indicadores, observou-se que as pessoas com até 59 anos chegam a superar os que têm 60 e mais, revelando uma proporção significativa de pessoas não idosas demandando cuidados complexos, principalmente nos grupos de maior exclusão. Exemplo disso são as menores razões idoso/não idoso observada entre os portadores de traqueostomia, alocados nos grupos com Exclusão alta, moderada e baixa, os usuários de sonda do grupo com Exclusão moderada e os portadores de úlceras por pressão no grupo com Exclusão alta.

Tabela 2 - Razão idoso/não idoso* para cada indicador de complexidade de cuidado e grupo - Município de São Paulo - 2003

\begin{tabular}{|c|c|c|c|c|}
\hline \multirow{2}{*}{$\begin{array}{l}\text { Indicador de complexidade de } \\
\text { cuidado }\end{array}$} & \multicolumn{4}{|c|}{ Grupos } \\
\hline & $\begin{array}{c}\text { Exclusão } \\
\text { Alta }\end{array}$ & $\begin{array}{c}\text { Exclusão } \\
\text { Moderada }\end{array}$ & $\begin{array}{c}\text { Exclusão } \\
\text { baixa }\end{array}$ & Exclusão muito baixa \\
\hline Oxigenoterapia & 1,78 & 1,71 & 1,88 & 5,00 \\
\hline Úlcera por pressão & 0,93 & 1,58 & 4,17 & 3,50 \\
\hline Sonda & 1,86 & 0,86 & 2,29 & $0 * *$ \\
\hline Traqueostomia & 0,88 & 0,85 & 0,79 & $0 * *$ \\
\hline
\end{tabular}

*obtida dividindo-se o somatório de pessoas com 60 anos e mais pelo somatório de pessoas de um a 59 anos, que apresentavam cada indicador de complexidade, por grupo; ** não havia pessoas com menos de 60 anos, impossibilitando o cálculo. 
Quando se analisa a distribuição das pessoas que apresentaram algum tipo de indicador de complexidade de cuidado e seu respectivo grau de incapacidade, verifica-se que o grau cinco, de mais severa incapacidade, concentra a maioria dos indicadores. Porém, a distribuição entre as escalas um, dois, três e quatro não permite afirmar que exista uma relação direta entre o grau de incapacidade e a complexidade de cuidado. O cálculo da razão de incapacidade, para cada indicador de complexidade de cuidado e grupo, mostra que as pessoas com úlcera por pressão e que fazem uso de sonda apresentam severa incapacidade em todos os grupos, principalmente nos dois de maior exclusão.

Tabela 3 - Razão de incapacidade* para cada indicador de complexidade de cuidado e grupo. Dados brutos - Município de São Paulo - 2003

\begin{tabular}{|c|c|c|c|c|}
\hline \multirow{2}{*}{$\begin{array}{l}\text { Indicador de complexidade de } \\
\text { cuidado }\end{array}$} & \multicolumn{4}{|c|}{ Grupos } \\
\hline & $\begin{array}{c}\text { Exclusão } \\
\text { Alta }\end{array}$ & $\begin{array}{c}\text { Exclusão } \\
\text { Moderada }\end{array}$ & $\begin{array}{c}\text { Exclusão } \\
\text { baixa }\end{array}$ & $\begin{array}{c}\text { Exclusão } \\
\text { muito baixa }\end{array}$ \\
\hline Oxigenoterapia & 0,47 & 0,78 & 1,88 & 1,00 \\
\hline Úlcera por pressão & 5,75 & 3,45 & 1,66 & 2,00 \\
\hline Sonda & 3,00 & 6,80 & 2,83 & 3,00 \\
\hline Traqueostomia & 1,14 & 1,40 & 1,08 & $0 * *$ \\
\hline
\end{tabular}

*obtida dividindo-se o somatório de pessoas com as graus 4 e 5 pelo somatório de pessoas com as graus 1, 2 e 3, que apresentavam cada indicador de complexidade, por grupo. **não havia pessoas com menos de 60 anos, impossibilitando o cálculo.

Os grupos de maior exclusão têm maior proporção de homens com menos de 60 anos com incapacidade severa e maior complexidade de cuidado, sendo o AVC a doença predominante. Já os grupos com menor exclusão apresentam proporcionalmente mais mulheres idosas com incapacidade leve e moderada e menor complexidade de cuidado, sendo o AVC e a hipertensão as doenças predominantes.

Em síntese, os grupos com Exclusão alta e moderada têm em média mais pacientes, maior proporção de homens com menos de 60 anos com incapacidade severa e que demandam cuidados de maior complexidade, sendo o AVC a doença predominante. Os grupos com Exclusão baixa e muito baixa têm proporcionalmente mais mulheres idosas com incapacidade leve e moderada e que requerem cuidados de menor complexidade, sendo o AVC e a hipertensão as patologias predominantes.

\section{DISCUSSÃO}

No município de São Paulo, dada a diversidade de realidades dos diferentes territórios, o provimento de cuidados de saúde no domicílio assume contornos específicos. Uma breve análise da dimensão estrutural, composta pelas políticas de saúde em âmbito nacional, em particular aquelas voltadas aos idosos e deficientes, faz perceber que as transições demográfica e epidemiológica, aliadas às políticas de ajuste vigentes no Brasil desde a década de 90, resultam não apenas no aumento de doenças crônico-degenerativas, mas também na piora nas condições de vida de importante parcela da população. No Brasil, a expansão das forças produtivas, a proletarização e a marginalização dos excluídos pelo sistema capitalista produziu um enorme contingente de excluídos e um crescimento assustador da violência urbana.
Àqueles que sobrevivem à violência cotidiana nas grandes cidades por vezes carregam algo mais que cicatrizes. As perdas funcionais e a dependência, freqüentemente associadas ao envelhecimento populacional, também podem resultar de seqüelas causadas pelas condições violentas de vida. Ao mesmo tempo em que se observa o crescimento da morbidade a partir dos 45 anos, atingindo 32,3\% das internações de pessoas com 80 e mais anos de idade, entre 600 e 700 mil pessoas são internadas por ano em hospitais da rede SUS em conseqüência de lesões por causas externas ${ }^{(19-20)}$. Essas pessoas muitas vezes acabam necessitando de internações prolongadas, com alto custo para o sistema de saúde. Os cuidados domiciliários no âmbito do SUS esboçadas até o momento são direcionadas para alguns grupos populacionais, em resposta à demanda dos serviços de saúde, não representando necessariamente uma resposta às necessidades sociais e de saúde da população, que sequer foram objeto de estudo específico.

Na dimensão particular, que compreende a descrição da situacionalidade, dinamicidade e historicidade do processo saúde-doença dos diferentes grupos sociais em diferentes distritos administrativos do município de São Paulo em relação à inclusão/ exclusão social, apesar do aumento da longevidade, evidencia-se alta morbimortalidade por causas externas em não idosos, resultando mais uma vez em aumento no número de pessoas com perdas funcionais por doenças próprias do envelhecimento ou resultantes das condições violentas de vida.

No presente estudo, as Lesões, envenenamentos e algumas outras conseqüências de causas externas responderam pelo atendimento domiciliário de 393 pessoas. Dessas, 200 (50,9\%) eram idosos acometidos principalmente por fratura de fêmur, mas 193 (49,1\%) tinham menos de 60 anos, 
vitimados predominantemente por Outros efeitos e os efeitos não especificados de causas externas.

Com o acréscimo no número de internações de pacientes com perdas funcionais, há sobrecarga dos caros serviços de internação hospitalares. Em que pesem as dificuldades em cuidar em domicílio de uma pessoa com limitações físicas temporárias ou permanentes, o alto custo de manter internado um paciente que pode ter seguimento ambulatorial fala em favor da necessidade de criação de serviços de assistência domiciliária não necessariamente vinculados aos hospitais, mas articulados a esses por meio de um sistema eficaz de referência e contra-referência.

Os programas de cuidados domiciliários existentes no município de São Paulo, alguns no âmbito da Atenção Básica, ainda atuam de forma desarticulada, não sendo capazes de assegurar a integralidade e a continuidade do cuidado. A falta de uma política municipal de assistência domiciliária capaz de subsidiar a estruturação dos serviços resulta em situações de iniqüidade em saúde. Na ausência de uma política pública claramente definida, o dilema de estabelecer quais as populações prioritárias para atendimento em domicílio acaba ficando nas mãos do profissional de saúde.

Na dimensão singular, que diz respeito aos perfis das pessoas que necessitam de cuidados em domicílio, observa-se a predominância de idosos (75\%), especialmente mulheres: $60 \%$ do total de pacientes são do sexo feminino e, dessas, $44 \%$ são idosas. As maiores diferenças em relação ao sexo se fazem notar a partir dos 75 anos, quando se observa decréscimo paulatino do número de homens. A partir dos 85 anos, há cerca de cinco mulheres idosas para cada homem na mesma faixa etária. Porém, quando se analisa este grupo de pessoas em relação ao Índice de exclusão, nota-se que, quanto maior a exclusão social, maior a proporção de pessoas com menos de 60 anos, especialmente do sexo masculino. Abaixo dos 40 anos, os homens são maioria.

\section{CONCLUSÕES}

As unidades de saúde do município de São Paulo ainda não estão estruturadas para responder às necessidades de saúde de pessoas com perdas funcionais e dependência que demandam por cuidados domiciliários. O mapeamento das pessoas com deficiência, incapacidade e dependência ainda é precário, dificultando o estabelecimento de políticas de saúde adequadas.

A Classificação Internacional de Funcionalidade, Incapacidade e Saúde (CIF) ${ }^{(21)}$ pode ser uma ferramenta importante no mapeamento das pessoas com perdas funcionais e dependência, já que não privilegia nenhum gênero ou grupo etário, além de apresentar uma concepção ampliada do processo saúde-doença, permitindo, se aliada a outras metodologias, um desenho aproximado da determinação social desse processo saúde-doença.

Para o dimensionamento de horas de enfermagem no domicílio, faz-se necessário aplicar um instrumento específico. Alguns autores recomendam o uso do Therapeutic Intervention Scoring System Intermediate - TISS-Intermediário ${ }^{(22)}$ que, apesar de mensurar horas de enfermagem considerando a complexidade de cuidado do paciente apenas no momento da alta hospitalar, pode ser uma ferramenta útil para o dimensionamento de profissionais de enfermagem em domicílio.

A característica de aproximação dos serviços de saúde ao cidadão traduzidos na capital paulista pela implantação do PSF nas áreas mais necessitadas representa, sem sombra de dúvida, a oportunidade de um acesso mais efetivo e de maior resposta às necessidades de saúde da população mais excluída.

O PSF pode representar uma estratégia eficiente frente ao desafio de atender esse contingente de pessoas que estão (sobre)vivendo com perdas funcionais e dependência, cujas necessidades são negligenciadas em função da falta de oportunidade e possibilidade em buscar atendimento em outros serviços, seja por dificuldade de mobilidade e/ ou locomoção, seja pela oferta ainda insuficiente de serviços de saúde que dêem conta deste perfil populacional.

Em São Paulo, é premente incorporar ao PSF a discussão sobre o cuidado domiciliário a pessoas com perdas funcionais e dependência, de modo a atender as especificidades do município, incluindo a definição de critérios que contemplem a diversidade dos distritos administrativos e os diferentes perfis saúde-doença, além da previsão de financiamento das ações e estabelecimento de uma rede articulada de cuidados, em que a Atenção Básica tem um papel fundamental.

\section{REFERÊNCIAS}

1. Carvalho JAM, Garcia RA. O envelhecimento da população brasileira: um enfoque demográfico. Cad Saúde Pública. 2003; 19(3):725-33.

2. Chaimowicz F. A saúde dos idosos brasileiros às vésperas do século XXI: problemas, projeções e alternativas. Rev Saúde Pública. 1997;31(2):184-200.
3. Nunes ATGL. Serviço social e universidade de terceira idade: uma proposta de participação social e cidadania para os idosos. Textos Envelhecimento [periódico na Internet].2001[citado 2006 jun. 20];3(5):[cerca de 7 p.] Disponível em: http:// www. unati. uerj.br/tse scielo. php? script=sci_arttex t\&pid= S1517 59282001000100003\&lng=pt\&nrm=iso 
4. Pereira APP. Formação em serviço social, política social e o fenômeno do envelhecimento. In: Mesa Redonda do Seminário sobre Educação Superior e Envelhecimento Populacional no Brasil; 2005 maio 11-12; Brasília, BR [evento na Internet]. Brasília: MEC; 2005. [citado 2006 jun. 28]. Disponível em: http://portal.mec.gov.br/sesu/arquivos/pdf/envelhecimento/ formacaosocialpotyara.pdf

5. Camargos MCS, Perpétuo IHO, Machado CJ. Expectativa de vida com incapacidade funcional em idosos em São Paulo, Brasil. Rev Panam Salud Publica [periódico na Internet]. 2005 [citado 2006 jun. 28];17(5/6):[cerca de 8 p.]. Disponível em: http:// www.scielosp.org/pdf/rpsp/v17n5-6/26275.pdf

6. Laurenti R, Mello Jorge MHP, Gotlieb SLD. Perfil epidemiológico da morbi-mortalidade masculina. Ciênc Saúde Coletiva. 2005:10(1):35-46.

7. Ramos LR, Rosa TEC, Oliveira ZMA, Medina MCG, Santos FRG. Perfil do idoso em área metropolitana na região sudeste do Brasil: resultados de inquérito domiciliar. Rev Saúde Pública. 1993;27(2):87-94.

8. Barreto KML, Carvalho EM, Falcão IV, Lessa FJD, Leite VMM. Perfil sócio-epidemiológico demográfico das mulheres idosas da Universidade Aberta à Terceira Idade no estado de Pernambuco. Rev Bras Saúde Mater Infan. 2003;3(3):339-54.

9. Laurell AS. A saúde-doença como processo social. In: Nunes ED, organizador. Medicina social: aspectos históricos e teóricos: São Paulo: Global; 1983. p. 133-58.

10. Santos TS. Globalização e exclusão: a dialética da mundialização do capital. Sociologias. 2001;(6):170-98.

11. Soares LTR. Globalização e exclusão: o papel social do enfermeiro. Esc Anna Nery Rev Enferm. 1997;1(1):13-21.

12. Ribeiro M. Exclusão: problematizando o conceito. Educ Pesq. 1999;25(1):35-49.

13. Pochmann M, organizador. Outra cidade é possível: alternativas de inclusão social em São Paulo. São Paulo: Cortez; 2003.
14. Campos A, Pochmann M, Amorim R, Silva R, coordenadores. Atlas da exclusão social no Brasil: dinâmica e manifestação territorial. São Paulo: Cortez; 2003. v. 2.

15. Sportello EF. Caracterização das formas de vida e trabalho das cuidadoras familiares do Programa de Assistência Domiciliária do Hospital Universitário da Universidade de São Paulo [dissertação]. São Paulo: Escola de Enfermagem, Universidade de São Paulo; 2002.

16. Coelho Filho JM. Modelos de serviços hospitalares para casos agudos em idosos. Rev Saúde Pública. 2000;34(6):666-71.

17. Organização Mundial da Saúde (OMS). CID 10 - Classificação Estatística Internacional de Doenças e problemas relacionados à saúde: $10^{\mathrm{a}}$ rev. São Paulo: Centro Colaborador da Organização Mundial da Saúde para a Classificação de Doenças em Português; 1995.

18. Egry EY. Saúde coletiva: construindo um novo método em enfermagem. São Paulo: Ícone; 1996.

19. Mello Jorge MHP, Yunes J. Violência e saúde no Brasil. Rev USP. 2001;(51):114-27.

20. Kilsztajn S, Rossbach A, Câmara MB, Carmo MSN. Serviços de Saúde, gastos e envelhecimento da população brasileira. In: $13^{\circ}$ Encontro da Associação Brasileira de Estudos Populacionais, 2002; Ouro Preto, BR [evento na Internet]. [citado 2006 jun. 28]. Disponível em: http://www.abep.nepo.unicamp.br/docs/ anais/pdf/2002/Com_ENV_ST31_Kilsztajn_texto.pdf

21. Organização Mundial da Saúde (OMS). Classificação Internacional de Funcionalidade, Incapacidade e Saúde. São Paulo: Centro Colaborador da Organização Mundial da Saúde para a Família de Classificações Internacionais/EDUSP; 2003.

22. Dal Ben LW, Sousa RMC. Adaptação de instrumento para dimensionar horas diárias de assistência de enfermagem residencial. Rev Esc Enferm USP. 2004;39(1):80-9. 